\title{
BMJ Open Carotid endarterectomy with primary closure versus patch angioplasty in patients with symptomatic and significant stenosis: protocol for a systematic review with meta-analyses and trial sequential analysis of randomised clinical trials
}

\author{
Martijn S Marsman, ${ }^{1}$ Jørn Wetterslev, ${ }^{2}$ Abdelkarime Khodadade Jahrome, ${ }^{1}$ \\ Christian Gluud, ${ }^{2}$ Frans L Moll, ${ }^{3}$ Amine Karimi, ${ }^{4}$ Frederik Keus, ${ }^{5}$ Giel G Koning ${ }^{1}$
}

To cite: Marsman MS, Wetterslev J, Jahrome AK, et al. Carotid endarterectomy with primary closure versus patch angioplasty in patients with symptomatic and significant stenosis: protocol for a systematic review with metaanalyses and trial sequential analysis of randomised clinical trials. BMJ Open 2019;9:e026419. doi:10.1136/ bmjopen-2018-026419

\section{- Prepublication history and} additional material for this paper are available online. To view these files, please visit the journal online (http://dx.doi. org/10.1136/bmjopen-2018026419).

Received 8 September 2018 Revised 24 February 2019 Accepted 27 February 2019

Check for updates

(C) Author(s) (or their employer(s)) 2019. Re-use permitted under CC BY-NC. No commercial re-use. See rights and permissions. Published by BMJ.

For numbered affiliations see end of article.

Correspondence to Martijn S Marsman; martijnmarsman@gmail.com

\section{ABSTRACT}

Introduction Use of patch angioplasty in carotid endarterectomy (CEA) is suggested to reduce the risk of restenosis and recurrent ipsilateral stroke. The objective is to conduct a systematic review with metaanalysis and trial sequential analysis as well as Grading of Recommendations Assessment, Development and Evaluation (GRADE) assessments comparing the benefits and harms of CEA with primary closure of the arterial wall versus CEA with patch angioplasty in patients with a symptomatic and significant carotid stenosis.

Methods and analysis The review shall be conducted according to this published protocol following the recommendations of the 'Cochrane' and reported according to the Preferred Reporting Items for Systematic Reviews and Meta-Analyses statement. Randomised clinical trials comparing CEA with primary closure of the arterial wall versus CEA with patch angioplasty (regardless of used patch materials) in human adults with a symptomatic and significant carotid stenosis will be included. Primary outcomes are all-cause mortality at maximal follow-up, health-related quality of life and serious adverse events. Secondary outcomes are symptomatic or asymptomatic arterial occlusion or restenosis, and non-serious adverse events. We will primarily base our conclusions on meta-analyses of trials with overall low risk of bias. However, if pooled point estimates of all trials are similar to pooled point estimates of trials with overall low risk of bias and there is lack of a statistical significant interaction between estimates from trials with overall high risk of bias and trials with overall low risk of bias we will consider the precision achieved in all trials as the result of our meta-analyses.

Ethics and dissemination The proposed systematic review will collect and analyse secondary data from published studies therefor ethical approval is not required. The results of the systematic review will be disseminated by publication in a peer-review journal and submitted for presentation at relevant conferences.
Strengths and limitations of this study

- The review shall be conducted according to this published protocol following the recommendations of the 'Cochrane' and reported according to the Preferred Reporting Items for Systematic Reviews and Meta-Analyses statement.

- Trial sequential analysis compared with GRADE assessments of randomised clinical trials are included.

- This review benefits from a comprehensive search strategy, designed to retrieve a broad spectrum of relevant articles for the research question.

- To avoid design error, one technique will be compared with one other technique.

PROSPERO registration number CRD42014013416.

\section{INTRODUCTION}

Carotid artery stenosis occurs due to atherosclerosis and was described to be a pathological substrate for ischaemic diseases of the ipsilateral brain and eye by C Miller Fisher in $1951 .^{1}$ Preventive management of asymptomatic carotid artery stenosis includes antiplatelets, statins, antihypertensives, diabetic control as well as lifestyle modifications. ${ }^{2-4}$ There is still discussion about the severity of the stenosis for surgical treatment and the way the severity of the stenosis should be assessed. Carotid endarterectomy (CEA) is the preferred treatment for patients with a symptomatic and significant $(>70 \%)$ stenosis of the carotid artery, ${ }^{5}$ primarily based on the European Carotid Surgery Trial and the 
North American Symptomatic Carotid Endarterectomy Trial. $^{6-8}$

Restenosis after CEA occurs in $6 \%-36 \%$ of patients during long-term follow-up of at least 12 months. ${ }^{9-13}$ Two operation techniques are well known in literature: the eversion technique and the traditional endarterectomy using a longitudinal arteriotomy. Closure in both techniques can be achieved by either direct suturing of the arterial wall or patch angioplasty in CEA. ${ }^{14}$ Use of patch angioplasty in CEA is suggested to reduce both the risks of restenosis and recurrent ipsilateral stroke. ${ }^{15}$

Guidelines of both the European Society of Vascular Surgery and the Dutch Society for Vascular Surgery consider CEA with patch angioplasty as the reference technique. ${ }^{81617}$ A meta-analysis of 10 randomised clinical trials (RCTs) including 2157 operations in 1967 patients compared CEA with primary closure versus CEA with patch angioplasty and concluded that CEA with patch angioplasty may reduce the risks of restenosis, perioperative arterial occlusion and ipsilateral stroke. ${ }^{15}$ However, the observed differences in intervention effects may be explained by several confounding factors and/or differential use of cointerventions, such as the use of perioperative transcranial Doppler monitoring, perioperative carotid pressure measurement, electroencephalographic monitoring, selected use of shunting, regional anaesthesia and variations in materials used for patching. ${ }^{18-25}$

To determine which technique, CEA with a primary closure of the arterial wall or CEA with use of patch angioplasty is more effective for a symptomatic and significant $(>70 \%)$ carotid stenosis, it is important that all available evidence is evaluated according to the risks of errors in a systematic review in line with the Cochrane Handbook for Systematic Reviews of Interventions. ${ }^{26}{ }^{27}$ Therefore, a proper and updated systematic review with meta-analyses and Trial Sequential Analysis (TSA) is needed, including GRADE assessments of the evidence.

\section{Objective}

The objective is to conduct a systematic review with meta-analysis and TSA of RCTs, evaluating the benefits and harms of the primary closure versus patch angioplasty in CEA according to a prepublished protocol following the Cochrane Handbook for Systematic Reviews of Interventions. ${ }^{26}$

\section{METHODS}

This review will be conducted according to this protocol which is also registered at PROSPERO since 2014 and was updated at October 2018 (http://www.crd.york.ac.uk/ PROSPERO/display_record.php?ID=CRD42014013416) following the recommendations of the "Cochrane Handbook for Systematic Reviews of interventions ${ }^{26}$ and will be reported according to the Preferred Reporting Items for Systematic Reviews and Meta-Analyses statement (at: www.prisma-statement.org). ${ }^{28}$

\section{Studies}

Only RCTs comparing CEA with primary closure of the arterial wall versus CEA with patch angioplasty (regardless of used patch materials) will be included. Trials will be considered irrespective of language, blinding, outcomes or publication status. We will also consider quasi-randomised studies, controlled clinical studies and other observational studies for data on harm if retrieved with our searches for RCTs. This is because adverse events are rarely reported in RCTs. ${ }^{29}$ Moreover, such observational studies may provide information on rare or late occurring adverse events. ${ }^{29}$ We are aware that the decision not to search for all observational studies may bias our review towards assessment of benefits and may overlook certain harms, such as late or rare harms.

\section{Patients}

According to the current guidelines, ${ }^{6-8}$ patients with a symptomatic and significant stenosis $(>70 \%$, measured by computed tomographic angiography or magnetic resonance angiography) of the carotid artery will be considered. Repeated Doppler ultrasound or digital subtraction angiography is possible as an imaging modality to measure the degree of the carotid stenosis, but the threshold of stenosis should be at least $70 \%$. Only trials which evaluate CEA in adult patients ( $\geq 18$ years) will be included. ${ }^{17}$ We are aware of the guideline statement that CEA may be considered in symptomatic internal carotid lesions of $<70 \%$. Studies in children and animals will be excluded.

\section{Experimental intervention}

The experimental intervention is traditional CEA (longitudinal arteriotomy) with primary closure of the arterial wall. ${ }^{14}$ RCTs which compare the eversion technique with patch angioplasty will be excluded. ${ }^{30}$ Because of comparing two techniques, the eversion technique will be investigated in a separate systematic review, we want to compare one experimental intervention to one control intervention to prevent design error.

\section{Control intervention}

The control intervention is CEA with patch angioplasty regardless of the type of patch material used..$^{14}$

\section{Cointerventions}

Intraoperative monitoring may vary in the trials such as the use of perioperative transcranial Doppler monitoring, perioperative carotid pressure measurement and electroencephalographic monitoring. Other intraoperative cointerventions may also vary in the trials, for example, the selected use of shunting and the use of variations in materials used for patching.

\section{Outcomes}

The outcome measures will be graded from the patients' perspective (GRADE Working Group 2008, online supplementary appendix figure 1). ${ }^{31}$ 


\section{Primary outcomes}

- All-cause mortality.

- Proportion of participants with one or more serious adverse events; that is, any untoward medical occurrence that results in death, is life-threatening, requires hospitalisation or prolongation of existing hospitalisation, results in persistent or significant disability or incapacity. $^{32}$

- Health-related quality of life: any scale used by trialists to assess the participants' reporting of their quality of life.

\section{Secondary outcomes}

- Symptomatic or asymptomatic (50\%-99\%) arterial occlusion or restenosis.

- Proportion of participants with one or more non-serious adverse events: any untoward medical occurrence in a participant who does not meet the above criteria for a serious adverse event is defined as a non-serious adverse event. ${ }^{32}$

\section{Exploratory outcomes}

- Separately reported serious adverse events.

- Separately reported non-serious adverse events.

The numbers of patients with one or more complications will be evaluated rather than the numbers of events, depending on the availability of data.

\section{Search strategy}

The Cochrane Central Register of Controlled Trials in The Cochrane Library, PubMed/MEDLINE and EMBASE will be searched. References of the identified trials will be searched to identify any further relevant RCTs. The search strategies are provided in online supplementary appendix 2. Searches will include MeSH descriptors such as 'Clinical Trials', 'carotid endarterectomy', 'thromboendarterectomy' and 'carotid artery disease'. We will also search online trial registries such as ClinicalTrials.gov (https:// clinicaltrials.gov/), European Medicines Agency (www. ema.europa.eu/ema/), WHO International Clinical Trial Registry Platform (www.who.int/ictrp) and the Food and Drug Administration (www.fda.gov) for ongoing or unpublished trials. In addition, we plan to search Google Scholar (https://scholar.google.nl/) using the term carotid endarterectomy in title.

\section{Data collection}

Two authors will perform screening and select the trials for inclusion, independently. Excluded trials and studies will be listed with their reasons for exclusion. While disagreements may occur, a third author will be approached to reconcile. The authors will extract the following data: trial characteristics (year and language of publication, country in which the trial was conducted, year of conduction of the trial, single or multicenter trial, number of patients), patient characteristics (inclusion and exclusion criteria, mean age, mean body mass index and gender, smoking, diabetes mellitus, use of statin and platelet inhibitors), intervention characteristics (primary closure, closure by patch, use of shunting), cointerventions (local or general anaesthesia, perioperative transcranial Doppler monitoring, perioperative carotid pressure measurement, electroencephalographic monitoring) and the outcome measures evaluated.

If there are any unclear or missing data, the corresponding authors of the individual trials will be contacted at least twice.

\section{Risk of bias assessment}

Two authors will assess the risks of bias, without masking for trial names, according to the Cochrane Handbook for Systematic Reviews of Interventions, ${ }^{26}$ including the domains of generation of the allocation sequence, allocation concealment, blinding of participants, personnel, and outcome assessors, incomplete outcome data, selective outcome reporting and other bias risks such as vested interests. Risk of bias components will be scored as low, unclear or high risk of bias. Trials will be classified as trials at low overall risk of bias if all risk of bias domains are scored as having low risk of bias. If one or more of the bias domains are scored as unclear or at high risk of bias, the trial will be considered at high overall risk of bias. ${ }^{27} 3334$

\section{Sequence generation}

- Low risk of bias: the method used (eg, central allocation) is unlikely to induce bias on the final observed effect, such as:

- Referring to a random number table.

- Using a computer random number generator.

- Coin tossing.

- Shuffling cards or envelopes.

- Throwing dice.

- Drawing of lots.

- Unclear risk of bias: insufficient information to assess whether the method used is likely to introduce confounders.

- High risk of bias: the method is improper and likely of introduce confounding, for example, based on date of admission, or record number, or by odd or even date of birth.

\section{Allocation concealment}

- Low risk of bias: participants and investigators enrolling participants could not foresee assignment because one of the following, or an equivalent method, was used to conceal allocation:

- Central allocation (including telephone).

- Web-based and pharmacy-controlled randomisation.

- Sequentially numbered drug containers of identical appearance.

- Sequentially numbered, opaque, sealed envelopes.

Unclear risk of bias: insufficient information to permit judgement of 'low risk' or 'high risk'. This is usually the case if the method of concealment is not described or not described in sufficient detail to allow a definite judgement. 
- High risk of bias: participants or investigators enrolling participants could possibly foresee assignments and thus introduce selection bias, such as allocation based on:

- An open random allocation schedule.

- Assignment envelopes were used without appropriate safeguards.

- Alternation or rotation.

- Date of birth.

- Case record number.

- Any other explicitly unconcealed procedure.

\section{Blinding of participants and personnel}

In surgical procedures, it is impossible to blind the surgeon who performs the procedure of CEA, while it is possible to blind the caregivers responsible for postoperative care as well as the patients. ${ }^{35}$ For this domain, we will consider the caregivers and patients and not the surgeon who performs the procedure, although a certain risk of bias will inevitably be present when evaluating surgical procedures. The statistician who performs the analyses can be blinded.

- Low risk of bias: no blinding or incomplete blinding, but the review authors judge that the outcome is not likely to be influenced by lack of blinding or blinding of participants and key study personnel ensured, and it is unlikely that the blinding could have been broken.

- Unclear risk of bias: insufficient information to permit judgement of low risk or high risk, or the study did not address this outcome.

- High risk of bias: no blinding or incomplete blinding, and the outcome is likely to be influenced by lack of blinding or blinding of key study participants and personnel attempted, but likely that the blinding could have been broken, and the outcome is likely to be influenced by lack of blinding.

\section{Blinding of outcome assessment}

- Low risk of bias: no blinding of outcome assessment, but the review authors judge that the outcome measurement is not likely to be influenced by lack of blinding or blinding of outcome assessment is ensured, and it is unlikely that the blinding could have been broken.

- Unclear risk of bias: insufficient information to permit judgement of low risk, or high risk or the study did not address this outcome.

- High risk of bias: no blinding of outcome assessment, and the outcome measurement is likely to be influenced by lack of blinding, or blinding of outcome assessment, but likely that the blinding could have been broken, and the outcome measurement is likely to be influenced by lack of blinding.

\section{Incomplete outcome data}

- Low risk of bias:

- No missing outcome data.
- Reasons for missing outcome data unlikely to be related to true outcome (for survival data, censoring unlikely to be introducing bias).

- Missing outcome data balanced in numbers across intervention groups, with similar reasons for missing data across groups.

- For dichotomous outcome data, the proportion of missing outcomes compared with observed event risk is not enough to have a clinically relevant impact on the intervention effect estimate.

- For continuous outcome data, plausible effect size (difference in means or standardised difference in means) among missing outcomes is not enough to have a clinically relevant impact on observed effect size.

- Missing data have been imputed using appropriate methods.

- Unclear risk of bias: insufficient reporting of attrition/exclusions to permit judgement of low risk or high risk (eg, number randomised not stated, no reasons for missing data provided) or the study did not address this outcome.

- High risk of bias:

- Reason for missing outcome data likely to be related to true outcome, with either imbalance in numbers or reasons for missing data across intervention groups.

- For dichotomous outcome data, the proportion of missing outcomes compared with observed event risk enough to induce clinically relevant bias in intervention effect estimate.

- For continuous outcome data, plausible effect size (difference in means or standardised difference in means) among missing outcomes enough to induce clinically relevant bias in observed effect size.

- 'As-treated' analysis done with substantial departure of the intervention received from that assigned at randomisation.

- Potentially inappropriate application of simple imputation.

\section{Selective outcome reporting}

- Low risk of bias: the study protocol is available and all the studies prespecified (primary and secondary) outcomes that are of interest in the review have been reported in the prespecified way, or the study protocol is not available but it is clear that the published reports include all expected outcomes, including those that were prespecified.

- Unclear risk of bias: insufficient information to permit judgement of low risk or high risk. It is likely that the majority of studies will fall into this category.

- High risk of bias:

- Not all of the studies prespecified primary outcomes have been reported.

- One or more primary outcomes is reported using measurements, analysis methods or subsets of the data (eg, subscales) that were not prespecified. 
- One or more reported primary outcomes were not prespecified (unless clear justification for their reporting is provided, such as an unexpected adverse effect).

- One or more outcomes of interest in the review are reported incompletely so that they cannot be entered in a meta-analysis.

- The study report fails to include results for a key outcome that would be expected to have been reported for such a study.

\section{Other bias}

- Low risk of bias: the study appears to be free of other sources of bias.

- Unclear risk of bias: there may be a risk of bias, but there is either insufficient information to assess whether an important risk of bias exists or insufficient rationale or evidence that an identified problem will introduce bias.

- High risk of bias: there is at least one important risk of bias.

\section{Statistical methods}

Meta-analyses will be performed according to the Cochrane Handbook for Systematic Reviews of Interventions. ${ }^{26}$ The software package Review Manager (RevMan) V.5.3 will be used. ${ }^{36}$ Significance levels will be adjusted due to multiplicity of several outcomes. The results of each outcome will be determinative for the use of the intervention and requires an adjusted statistical significance level (threshold). An alfa of $(0.05 /((1+3) / 2)=)$ 0.025 will be used for the primary outcomes to keep the family wise error rate $<0.05$. For the secondary outcomes, this will be $0.033 .^{3738}$ For exploratory outcomes, we will consider a $p$ value $<0.05$ as significant, because we view these outcomes as only hypothesis-generating outcomes. For dichotomous variables, the risk ratio with TSA-adjusted CIs will be calculated. For continuous variables, the mean difference $(\mathrm{MD})$ or the standardised mean difference with $95 \%$ CI will be calculated.

For the outcome of serious adverse events (SAE), we plan to estimate the proportion of patients with one or more SAE in each group and to analyse this outcome in a binary meta-analysis. However, as we anticipate the reporting of SAEs in trials to vary considerably we plan to do two analyses:

- Assuming that only one SAE is reported per patient we will add all reported SAE in each trial and calculate the proportion of summed SAE divided with number of randomised patients in the experimental and control intervention group (worst case scenario).

- To avoid multiple counts of SAE in the same patients (SAE counting is not a statistical independent outcome) we will also analyse the most frequent SAE as if it represents the total number of SAEs in the experimental and control intervention group (best case scenario). Being aware that none of these intervention effect estimates are exactly correct we will discuss them as possible worst and best case scenarios for the effect of the experimental versus the control intervention on the proportion of patients with one or more SAEs.

The impact of attrition bias will be explored using best/ worstand worst/bestcase scenarios: a best/worstcase scenario is one where all patients lost to follow-up in the intervention group are supposed to have survived while all patients lost to follow-up in the control intervention group have died. A worst/best case scenario is the reverse.

Heterogeneity will be explored by $\chi^{2}$ test with significance set at $p$ value of 0.10 , and the quantity of heterogeneity will be measured by $\mathrm{I}^{2}$. We will conduct both random-effects model and fixed-effect model meta-analyses. In case of discrepancies, the results of both models will be presented and we will primarily stress the result of the model with the result closet to null effect due to principle of cautiousness. ${ }^{38}$ The analyses will be performed on an intention-to-treat basis whenever possible.

A funnel plot will be used to explore small trial bias and to use asymmetry in funnel plot of trial size against treatment effect to assess this bias. Begg's and Egger's tests will be used to test for asymmetry in funnel plots. ${ }^{39}$

\section{Trial sequential analyses}

Meta-analyses may result in type I errors and type II errors due to an increased risk of random error when sparse data are analysed and due to repeated significance testing when a cumulative meta-analysis is updated with new trials. ${ }^{40}$ To assess the risk of type I and type II errors, TSA will be used. The vast majority of meta analyses $(\sim 80 \%)$ in Cochrane systematic reviews have less than the required information size to conclude on a $30 \%$ relative risk reduction (RRR) and $<2 \%$ have sufficient power to conclude on a $10 \%$ RRR. $^{42-44}$

TSA combines information size estimation for meta-analysis (cumulated sample size of included trials) with an adjusted threshold for statistical significance of meta-analysis. ${ }^{404145}$ The latter, called trial sequential monitoring boundaries (TSMBs), reduce type I errors. In TSA, the addition of each trial in a cumulative meta-analysis is regarded as an interim analysis and helps to clarify whether additional trials are needed or not. The idea in TSA is that when the cumulative z-curve crosses the TSMB, a sufficient level of evidence has been reached and no further trials may be needed. If the z-curve does not cross the boundary of benefit and the required information size has not been reached, there may be insufficient evidence to reach a conclusion. ${ }^{4041647}$ TSA can also be used for the evaluation of type II errors, that is, to evaluate whether further randomised trial is futile to show or discard the anticipated intervention effect (RRR or MD). This happens when the cumulative z-curve does cross the TSMBs for futility. TSA will be applied since it controls the risks of type I and type II errors in a cumulative meta-analysis and may provide important information on how many more patients need to be included in further trials. The information size will be calculated as 
diversity-adjusted required information size (DARIS). ${ }^{48}$ We will do the primary analysis calculating the DARIS based on an a priori anticipated intervention effect of a $10 \%$ RRR which is close to a minimal important difference and sensitivity analyses for a 15\% RRR as well as a the RRR suggested by the meta-analysis of the included trials. ${ }^{49}$ If the estimated diversity of the meta-analysis is $0 \%$, a sensitivity analysis with TSA using a diversity of $25 \%$ will be conducted. TSA will be performed on all outcomes. The required information size for primary outcomes will be calculated based on an a priori RRR of $10 \%$ and appropriately adjusted for diversity according to an overall type I error of 5\% and a power of $90 \%$ considering early and repetitive testing. ${ }^{48}$ For secondary outcomes, the DARIS will be calculated using a power of $80 \%{ }^{48}$

As a sensitivity analysis, the DARIS will be calculated using the estimated intervention effect from the trials at low risk of bias in a conventional meta-analysis. If the required information size is surpassed for the TSA using the estimated intervention effect in the conventional meta-analysis or a TSMB is crossed a TSA with an anticipated intervention effect equal to the confidence limit closest to the null effect in the effect estimate from the conventional meta-analysis will be performed. The TSAs will be conducted using the control event proportion calculated from the unweighted control event proportion from the control groups of the actual meta-analyses.

\section{Subgroup analyses}

The following subgroup analyses will be performed:

- trials at overall low risk of bias (all except blinding of surgeons scored as low risk of bias) compared with trials at high overall risk of bias (one or more of the bias domains [excluding blinding of surgeons] scored as unclear or high risk).

- different patch materials may be used including venous, polytetrafluorethylene, Dacron and biopatches (bovine/porcine). ${ }^{25}$ Subgroup analyses will be conducted according availability of data on different materials.

\section{GRADE}

Summary of findings tables will be produced summarising the results of the trials with overall low risk of bias and for all trials, separately. Reasons for downgrading the quality of the available evidence are risk of bias evaluation of the included bias domains, publication bias, heterogeneity, imprecision and indirectness (eg, length of stay is a surrogate outcome measure) ${ }^{50-52}$ We will compare the imprecision assessed according to GRADE with that of TSA. ${ }^{53}$

\section{Patient and public involvement}

Patients and/or public were not involved in this study.

\section{ETHICS AND DISSEMINATION}

The results of the systematic review will be disseminated by publication in a peer-review journal and submitted for presentation at relevant conferences.
Author affiliations

${ }^{1}$ Department of Vascular Surgery, HFG, Medical Center Leeuwarden, Leeuwarden, Netherlands

${ }^{2}$ The Copenhagen Trial Unit, Centre for Clinical Intervention Research, Copenhagen University Hospital Rigshospitalet, Copenhagen, Denmark

${ }^{3}$ Department of Vascular Surgery, University Medical Center Utrecht, Utrecht, Netherlands

${ }^{4}$ Department of Vascular Surgery, Rijnstate Hospital, Arnhem, Netherlands ${ }^{5}$ Department of Critical Care, University of Groningen, University Medical Center Groningen, Groningen, Netherlands

Acknowledgements The authors would like to thank Mrs WMT Peters, medical information specialist (Medical Library, Radboud University Nijmegen, the Netherlands) for her assistance. Also, we like to thank Mrs LWM Boerboom, MSc, medical information specialist (Medical Library, Elisabeth Tweesteden Hospital, Tilburg, the Netherlands) for her assistance.

Contributors MM is the first author of the protocol. MM, GGK and AK managed the first draft of this manuscript and coordinated the contributions of coauthors. Contributors JW, AKJ, CG, FLM, AK, FK and GGK contributed to the design of the study and revised the paper critically. JW, CG, FK and GGK provided professional and statistical support. All authors read and approved the final version of the manuscript. GGK was initiator and supervisor.

Funding The authors have not declared a specific grant for this research from any funding agency in the public, commercial or not-for-profit sectors.

Competing interests None declared.

Patient consent for publication Not required.

Ethics approval The proposed systematic review will collect and analyse secondary data from published studies therefore ethical approval is not required.

Provenance and peer review Not commissioned; externally peer reviewed.

Open access This is an open access article distributed in accordance with the Creative Commons Attribution Non Commercial (CC BY-NC 4.0) license, which permits others to distribute, remix, adapt, build upon this work non-commercially, and license their derivative works on different terms, provided the original work is properly cited, appropriate credit is given, any changes made indicated, and the use is non-commercial. See: http://creativecommons.org/licenses/by-nc/4.0/.

\section{REFERENCES}

1. Fisher M. Occlusion of the internal carotid artery. AMA Arch Neurol Psychiatry 1951;65:346-77.

2. Raman G, Moorthy D, Hadar N, et al. Management strategies for asymptomatic carotid stenosis: a systematic review and metaanalysis. Ann Intern Med 2013;158:676-85.

3. Abbott AL. Medical (nonsurgical) intervention alone is now best for prevention of stroke associated with asymptomatic severe carotid stenosis: results of a systematic review and analysis. Stroke 2009;40:e573-83.

4. Constantinou J, Jayia P, Hamilton G. Best evidence for medical therapy for carotid artery stenosis. J Vasc Surg 2013;58:1129-39.

5. Orrapin S, Rerkasem K. Carotid endarterectomy for symptomatic carotid stenosis. Cochrane Database Syst Rev 2017;6:CD001081.

6. Warlow C. MRC European Carotid Surgery Trial: interim results for symptomatic patients with severe $(70-99 \%)$ or with mild $(0-29 \%)$ carotid stenosis. The Lancet 1991;337:1235-43.

7. Barnett HJM, Taylor DW, Haynes RB, et al. Beneficial effect of carotid endarterectomy in symptomatic patients with high-grade carotid stenosis. N Engl J Med 1991;325:445-53.

8. Naylor AR, Ricco JB, de Borst GJ, et al. Editor's Choice Management of Atherosclerotic Carotid and Vertebral Artery Disease: 2017 Clinical Practice Guidelines of the European Society for Vascular Surgery (ESVS). Eur J Vasc Endovasc Surg 2018;55:3-81.

9. Bernstein EF, Torem S, Dilley RB. Does carotid restenosis predict an increased risk of late symptoms, stroke, or death? Ann Surg 1990;212:629-36.

10. Knudsen L, Sillesen H, Schroeder T, et al. Eight to ten years followup after carotid endarterectomy: clinical evaluation and Doppler examination of patients operated on between 1978-1980. Eur J Vasc Surg 1990;4:259-64.

11. Ouriel K, Green RM. Clinical and technical factors influencing recurrent carotid stenosis and occlusion after endarterectomy. J Vasc Surg 1987;5:702-6. 
12. Volteas $\mathrm{N}$, Labropoulos $\mathrm{N}$, Leon $\mathrm{M}$, et al. Risk factors associated with recurrent carotid stenosis. Int Angiol 1994;13:143-7.

13. Zierler RE, Bandyk DF, Thiele BL, et al. Carotid artery stenosis following endarterectomy. Arch Surg 1982;117:1408-15.

14. De Bakey ME, Crawford ES, Cooley DA, et al. Surgical considerations of occlusive disease of innominate, carotid, subclavian, and vertebral arteries. Ann Surg 1959;149:690-710.

15. Rerkasem K, Rothwell PM. Patch angioplasty versus primary closure for carotid endarterectomy. Cochrane Database Syst Rev 2009;4:CD000160.

16. Liapis CD, Bell PR, Mikhailidis D, et al. ESVS guidelines. Invasive treatment for carotid stenosis: indications, techniques. Eur J Vasc Endovasc Surg 2009;37(4 Suppl):1-19.

17. Nederlandse vereniging voor neurologie. Diagnostiek, behandeling en zorg voor patiënten met een beroerte. 2008 http://med-info.nl/ Richtlijnen/Geriatrie/Beroerte. pdf (Accessed 02 Dec 2017).

18. Bass A, Krupski WC, Schneider PA, et al. Intraoperative transcranial doppler: limitations of the method. J Vasc Surg 1989;10:549-53.

19. Gnanadev DA, Wang N, Comunale FL, et al. Carotid artery stump pressure: how reliable is it in predicting the need for a shunt? Ann Vasc Surg 1989;3:313-7.

20. Kresowik TF, Worsey MJ, Khoury MD, et al. Limitations of electroencephalographic monitoring in the detection of cerebral ischemia accompanying carotid endarterectomy. $J$ Vasc Surg 1991;13:439-43.

21. Kearse LA, Brown EN, McPeck K. Somatosensory evoked potentials sensitivity relative to electroencephalography for cerebral ischemia during carotid endarterectomy. Stroke 1992;23:498-505.

22. Benjamin ME, Silva MB, Watt $\mathrm{C}$, et al. Awake patient monitoring to determine the need for shunting during carotid endarterectomy. Surgery 1993;114:673-9.

23. Vaniyapong T, Chongruksut W, Rerkasem K. Local versus general anaesthesia for carotid endarterectomy. Cochrane Database Syst Rev 2013;4:CD000126.

24. Chongruksut W, Vaniyapong T, Rerkasem K. Routine or selective carotid artery shunting for carotid endarterectomy (and different methods of monitoring in selective shunting). Cochrane Database Syst Rev 2014;51:CD000190.

25. Rerkasem K, Rothwell PM. Patches of different types for carotid patch angioplasty. Cochrane Database Syst Rev 2010;24:CD000071.

26. In: Higgins JPT, Green S, eds. Cochrane Handbook for Systematic Review of Intervention Version 5.1.0 [updated March 2011]. 2011: The Cochrane Collaboration. http://www.Cochrane-handbook.org

27. Keus F, Wetterslev J, Gluud C, et al. Evidence at a glance: error matrix approach for overviewing available evidence. BMC Med Res Methodol 2010;10:90.

28. Moher D, Liberati A, Tetzlaff J, et al. Preferred reporting items for systematic reviews and meta-analyses: the prisma statement. PLoS Med 2009;6:e1000097.

29. Storebø OJ, Pedersen N, Ramstad E, et al. Methylphenidate for attention deficit hyperactivity disorder (ADHD) in children and adolescents - assessment of adverse events in non-randomised studies. Cochrane Database Syst Rev 2018;5:CD012069.

30. Cao PG, de Rango P, Zannetti S, et al. Eversion versus conventional carotid endarterectomy for preventing stroke. Cochrane Database Syst Rev 2001;125:CD001921.

31. Guyatt GH, Oxman AD, Kunz R, et al. What is "quality of evidence" and why is it important to clinicians? BMJ 2008;336:995-8.

32. International Conference on Harmonisation Expert Working Group. International conference on harmonization of technical requirements for registration of pharmaceuticals for human use. ICH harmonised tripartite guideline. Guideline for good clinical practice CFR \& ICH Guidelines. Vol. 1, Pennsylvania (PA): Barnett International/PAREXEL 1997.
33. Higgins J, Churchill R, Lasserson T, et al. Update from the Methodological Expectations of Cochrane Intervention Reviews (MECIR) project. Cochrane methods 2012:2-3.

34. Savović J, Turner RM, Mawdsley D, et al. Association Between Riskof-Bias Assessments and Results of Randomized Trials in Cochrane Reviews: The ROBES Meta-Epidemiologic Study. Am J Epidemiol 2018;187:1113-22.

35. Gurusamy KS, Gluud C, Nikolova D, et al. Assessment of risk of bias in randomized clinical trials in surgery. Br J Surg 2009;96:342-9.

36. Review Manager. RevMan) [Windows]. Version 5.3. Copenhagen: The Nordic Cochrane Centre, The Cochrane Collaboration, 2014.

37. Jakobsen JC, Wetterslev J, Lange T, et al. Viewpoint: taking into account risks of random errors when analysing multiple outcomes in systematic reviews. Cochrane Database Syst Rev 2016;3:2-7.

38. Jakobsen JC, Wetterslev J, Winkel P, et al. Thresholds for statistical and clinical significance in systematic reviews with meta-analytic methods. BMC Med Res Methodol 2014;14:1-13.

39. Koning GG, Wetterslev J, van Laarhoven CJHM, et al. The totally extraperitoneal method versus lichtenstein's technique for inguinal hernia repair: a systematic review with meta-analyses and trial sequential analyses of randomized clinical trials. PLoS One 2013;8:e52599.

40. Wetterslev J, Thorlund $\mathrm{K}$, Brok J, et al. Trial sequential analysis may establish when firm evidence is reached in cumulative meta-analysis. $J$ Clin Epidemiol 2008;61:64-75.

41. Brok J, Thorlund K, Wetterslev J, et al. Apparently conclusive metaanalyses may be inconclusive-Trial sequential analysis adjustment of random error risk due to repetitive testing of accumulating data in apparently conclusive neonatal meta-analyses. Int $\mathrm{J}$ Epidemiol 2009;38:287-98.

42. Turner RM, Bird SM, Higgins JPT. The impact of study size on metaanalyses: examination of underpowered studies in cochrane reviews. PLoS One 2013;8:e59202-8.

43. Mascha EJ. Alpha, beta, meta: guidelines for assessing power and type i error in meta-analyses. Anesth Analg 2015;121:1430-3.

44. Imberger G, Thorlund K, Gluud C, et al. False-positive findings in Cochrane meta-analyses with and without application of trial sequential analysis: an empirical review. BMJ Open 2016;6:e011890.

45. Thorlund K, Devereaux PJ, Wetterslev J, et al. Can trial sequential monitoring boundaries reduce spurious inferences from metaanalyses? Int J Epidemiol 2009;38:276-86.

46. Pogue J, Yusuf S. Overcoming the limitations of current metaanalysis of randomised controlled trials. The Lancet 1998;351:47-52.

47. Pogue JM, Yusuf S. Cumulating evidence from randomized trials: utilizing sequential monitoring boundaries for cumulative metaanalysis. Control Clin Trials 1997;18:580-93.

48. Wetterslev J, Thorlund K, Brok J, et al. Estimating required information size by quantifying diversity in random-effects model meta-analyses. BMC Med Res Methodol 2009;9:86.

49. Wetterslev J, Jakobsen JC, Gluud C. Trial Sequential Analysis in systematic reviews with meta-analysis. BMC Med Res Methodol 2017;17:1-18.

50. Guyatt GH, Oxman AD, Kunz R, et al. GRADE guidelines 6 . Rating the quality of evidence-imprecision. J Clin Epidemiol 2011;64:1283-93.

51. Savović J, Jones HE, Altman DG, et al. Influence of reported study design characteristics on intervention effect estimates from randomised controlled trials: combined analysis of metaepidemiological studies. Health Technol Assess 2012;16:1-82.

52. Savović J, Jones HE, Altman DG, et al. Influence of reported study design characteristics on intervention effect estimates from randomized, controlled trials. Ann Intern Med 2012;157:429-38.

53. Castellini G, Bruschettini M, Gianola S, et al. Assessing imprecision in Cochrane systematic reviews: a comparison of GRADE and Trial Sequential Analysis. Syst Rev 2018;7:110. 